\title{
Hysteroscopic adhesiolysis using blunt spreading dissection technique in severe intrauterine adhesions with false passage
}

\author{
Xiuting Zhu", Xingping Zhao", Xiangyang Zeng, Arvind Burjoo, Yimin Yang, Dabao Xu \\ Department of Obstetrics and Gynecology, Third Xiangya Hospital of Central South University, Changsha 410013, China \\ \#These authors contributed equally to this work. \\ Correspondence to: Yimin Yang, MD; Dabao Xu, MD. Department of Obstetrics and Gynecology, Third Xiangya Hospital of Central South University, \\ 138 Tongzipo Rd., Changsha 410013, China. Email: 492503562@qq.com; dabaoxu@yahoo.com.
}

Submitted Dec 27, 2019. Accepted for publication Jan 14, 2020.

doi: 10.21037/atm.2020.01.85

View this article at: http://dx.doi.org/10.21037/atm.2020.01.85

\section{Clinical vignette}

A 31 years old $\mathrm{G}_{3} \mathrm{P}_{1 \text { (C-Section) }} \mathrm{A}_{2}$ patient attended our hospital with a history of amenorrhea for 70 days following surgical termination of an $8 \mathrm{w} 4 \mathrm{~d}$ missed abortion with no cyclic lower abdominal pain. The patient had undergone two artificial cycle treatments after surgical termination, and there was no recovery with her menstruation. A hysteroscopy five days before this presentation in other hospital revealed severe intrauterine adhesions and a "tubular" uterine cavity. Pelvic examination revealed no abnormality. The urine pregnancy test was negative. An ultrasound scan revealed: C-Section scar, intrauterine adhesions and normal adnexa. The endometrium was marginally irregular, with uneven thickness and an interrupted III-line (Figure 1). Threedimensional (3D) color Doppler ultrasound showed the obliteration of the lower uterine segment with undetectable endometrium. The distance between the bilateral uterine horns was $18.3 \mathrm{~mm}$ (Figure 2). Preoperative diagnosis was "severe intrauterine adhesions (AFS score: 12)".

\section{Surgical techniques}

\section{Preparation}

The patient was taken to the operation room and adopted a lithotomy position. After routine disinfection and draping, intravenous anesthesia was successfully administered.

\section{Exposition}

Gynecological examination showed that the uterus was anteverted, and the cervix was exposed and disinfected.
Sterile saline solution was used to distend the uterus at a distension pressure of $120 \mathrm{mmHg}$, with a flow rate of $350 \mathrm{~mL} / \mathrm{min}$. Hysteroscopy was performed under ultrasound monitoring.

\section{Operation}

Hysteroscopy found that the internal os was closed with adhesions, and a normal uterine cavity could not be distinguished. At the same time, a suspected false passage could be seen, with an irregular morphology and a rough surface, leading to the lower-left uterine wall. An unidentified dark shadow hole could be seen in the upper-right of the false passage (Figure 3). The surgeon did not explore the false passage further and then used a 7-Fr double-action forceps to dissect and enlarge the hole gradually with blunt spreading dissection technique under direct hysteroscopic vision (1). After the dissection step by step, the right uterine fundus could be visualized. The left side of the uterine cavity was wholly obliterated, while the right side and middle-lower segment partially obliterated. After successful separation of these adhesions with a 7-Fr single action scissors (2), the whole uterine fundus could be seen. At this moment, the left fallopian tubal ostium still could not be seen because of the adhesions in the left uterine horn. Again, we use the 7-Fr single action scissors with blunt spreading dissection technique to dissect and separate the adhesions until the left fallopian tubal ostium is exposed. Postoperatively, the uterine cavity was normal, bilateral uterine horns and fallopian tubal ostia were visible at hysteroscopy (Figure 3). The uterine sounding was performed under ultrasound monitoring, and the uterine 

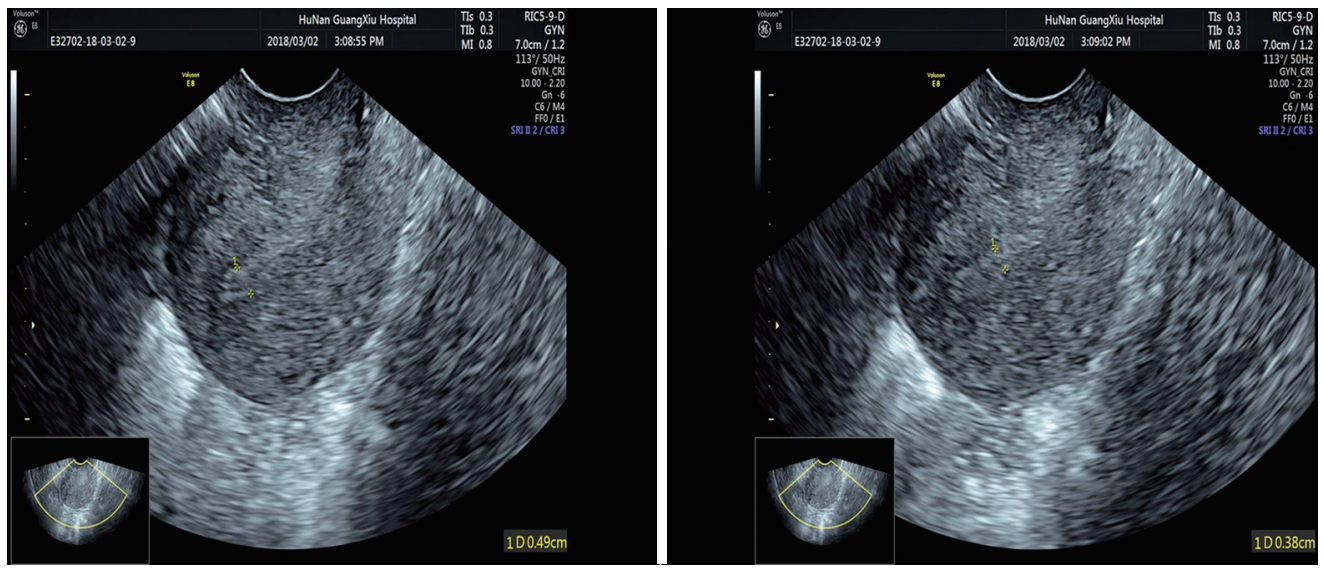

Figure 1 The endometrium was marginally irregular, with uneven thickness and an interrupted III-line.
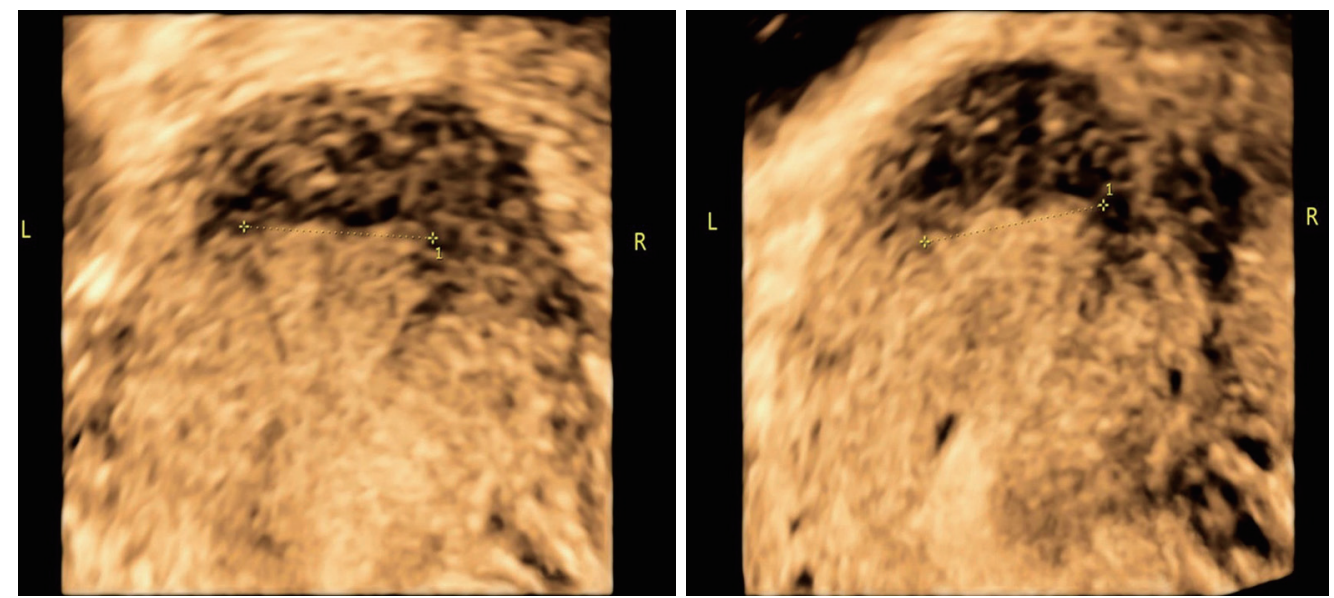

Figure 2 Three-dimensional (3D) color Doppler ultrasound showing the obliteration of the lower uterine segment with undetectable endometrium. The distance between the bilateral uterine horns was $18.3 \mathrm{~mm}$.

depth was $7.5 \mathrm{~cm}$. The cervix was gradually dilated with Hegar dilators up to number 8 bar, and in doing so, the lower and middle uterine segments were also expanded. According to the experience of the surgeon and the predetermined distance between bilateral uterine horns by preoperative ultrasound, the surgeon inserted a mediumsized intrauterine device (IUD) into the uterine cavity under ultrasound monitoring. The position of the IUD was checked via hysteroscopy to ensure that the size of the IUD matched the uterine cavity size, and the IUD was correctly positioned (Figure 3). The procedure of HA is shown in the Figure 4.

\section{Completion}

At the end of the operation, a 12 Fr-Foley catheter with its top part removed was inserted into the uterine cavity under ultrasound guidance, with the distal end directed towards the uterine fundus. The catheter balloon was then distended with $2.5 \mathrm{~mL}$ sterile saline. And then, $3 \mathrm{~mL}$ of the hyaluronic acid gel was injected through the other channel of Foley catheter into the uterine cavity. The indwelling Foley catheter was kept in situ for two weeks. Hormonal therapy was started with $3 \mathrm{mg}$ estradiol valerate Bid for 21 days and $200 \mathrm{mg}$ progesterone Qn for the last six days of the cycle. 

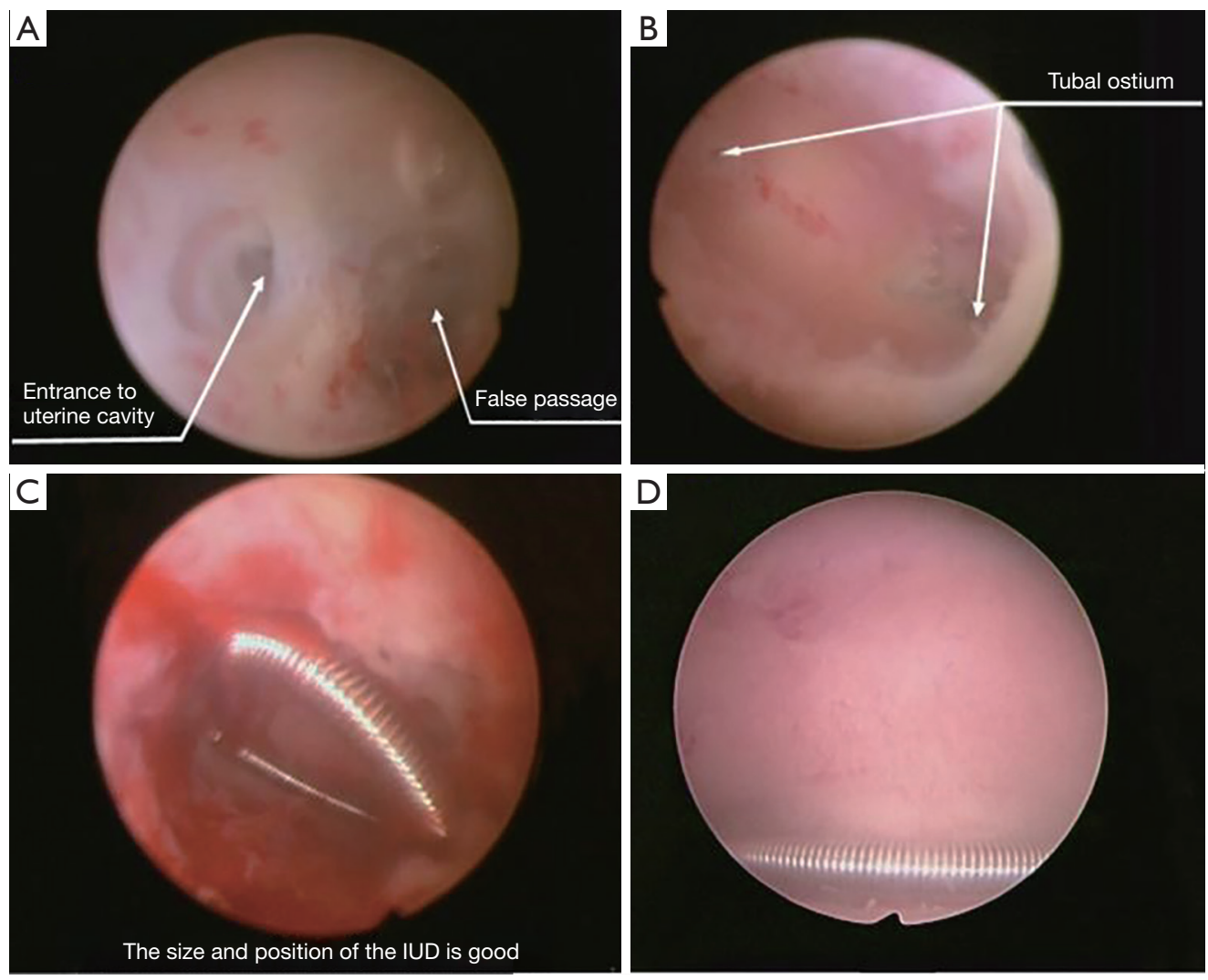

Figure 3 Hysteroscopic adhesiolysis. (A) Finding out the false passage and the correct channel entering the actual uterine cavity; (B) using double-action forceps to dissect intrauterine adhesions with blunt spreading dissection technique, and finally found the bilateral fallopian tubal ostia; (C) inserted a medium-sized intrauterine device into the uterus under ultrasonic monitoring; (D) postoperative follow-up hysteroscopy 1 month later revealed the uterine cavity was normal, the endometrium was dramatically better than before.

\section{Comments}

\section{Clinical results}

The operation was successfully conducted, and no complications occurred. The postoperative uterine cavity shape was normal, and there was no uterine perforation with the primary false passage. Postoperative follow-up hysteroscopy one month later revealed primary false passage was healed, uterine cavity shape was normal, the density of endometrium glands improved dramatically (Figure 3), IUD was correctly positioned and fallopian tubal ostia were visualized. The patient's menstruation returned to normal.

\section{Advantages}

Hysteroscopic adhesiolysis using blunt spreading dissection technique in severe intrauterine adhesions with the false passage is effective and safe, especially when the endometrial line is difficult to be identified by ultrasound monitoring. This technique can help improve the success rate of finding the correct intrauterine layer in such patients with intrauterine adhesions and reduce the probability of uterine perforation. In this case, the patient's lower segment endometrium of the uterine cavity was absent, and the endometrium could not be detected by ultrasound. Therefore, the blunt spreading dissection technique played a crucial role in finding and dissecting the actual uterine cavity of this patient. At the same time, if strayed into a muscular layer mistakenly, this technique can help discover and correct mistakes timely through muscular layer features including light pink color, latticed structure, and enriched in blood vessels, avoiding the occurrence of uterine perforation.

Moreover, this technique has no thermal or electrical injury. Additionally, if a slimmer hysteroscope with a 5-Fr working channel was available for this patient, we would 


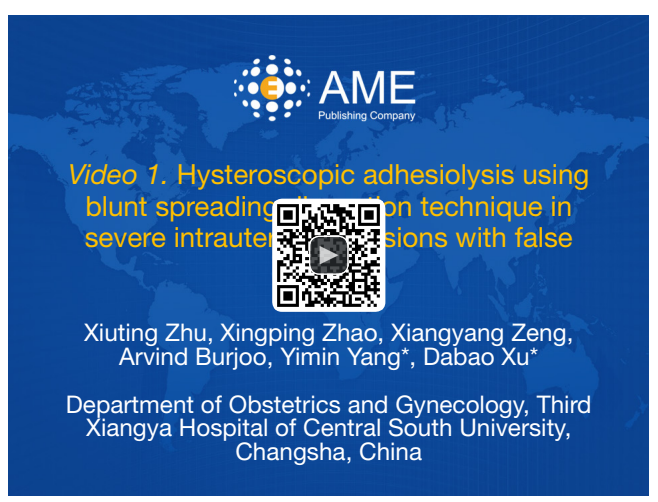

Figure 4 Hysteroscopic adhesiolysis using blunt spreading dissection technique in severe intrauterine adhesions with false passage (3). Available online: http://www.asvide.com/watch/33098

prefer the slimmer one. And for the hysteroscopists who are not very familiar with the use of the blunt spreading dissection technique, a double-action spoon-shaped forceps instead of the scissors is recommended to be used to expose the tubal ostium in the uterine horn.

\section{Caveats}

During hysteroscopic surgery, there may be many complications, such as bleeding and perforation. For those patients with earlier hysteroscopy or surgical history, it is particularly important to pay attention to whether there is a false passage in the muscular layer of the uterus. What's more, once finding a false passage in the uterine cavity, it is necessary to find the real uterine cavity rather than a false cavity and then separate the intrauterine adhesions further, to reduce the risk of uterine perforation. For those patients with severe intrauterine adhesions with the false passage, the use of double-action forceps during hysteroscopic surgery to find and expand the actual intrauterine cavity can improve the success rate of finding the correct intrauterine layer and reduce the probability of uterine perforation. This

Cite this article as: Zhu X, Zhao X, Zeng X, Burjoo A, Yang Y, $\mathrm{Xu}$ D. Hysteroscopic adhesiolysis using blunt spreading dissection technique in severe intrauterine adhesions with false passage. Ann Transl Med 2020;8(4):58. doi: 10.21037/ atm.2020.01.85 surgical technique is worthy of further clinical promotion.

\section{Acknowledgments}

Funding: This study was supported by the National Key Research and Development Program of China (Grant No. 2018YFC1004800) and the Natural Science Foundation of China (Grant No. 81671492).

\section{Footnote}

Conflicts of Interest: The authors have no conflicts of interest to declare.

Ethical Statement: The authors are accountable for all aspects of the work in ensuring that questions related to the accuracy or integrity of any part of the work are appropriately investigated and resolved. Approval was given to the study by The Institutional Review Board (IRB) of Third Xiangya Hospital and Xiangya Hospital, Central South University. The procedure was performed by relevant guidelines and regulations. Informed consent was obtained after the procedure was fully explained by all participants and their legal guardians.

\section{References}

1. Huang H, Cheng C, Johnson G, et al. Hysteroscopic Intrauterine Adhesiolysis Using a Blunt Spreading Dissection Technique With Double-action Forceps. J Minim Invasive Gynecol 2018;25:583-4.

2. Zhang A, Jamail G, Xue M, et al. Hysteroscopic Intrauterine Adhesiolysis Using the "Ploughing" Technique With Cold Scissors. J Minim Invasive Gynecol 2015;22:934-5.

3. Zhu X, Zhao X, Zeng X, et al. Hysteroscopic adhesiolysis using blunt spreading dissection technique in severe intrauterine adhesions with false passage. Asvide 2020;7:058. Available online: http://www.asvide.com/watch/33098 